\title{
EL INGLÉS EN EUROPA: ORIGEN Y CONSOLIDACIÓN DE UNA LENGUA FRANCA ${ }^{1}$
}

\author{
David Fernández Vítores ${ }^{2}$
}

\begin{abstract}
Resumen: Aunque la enseñanza del inglés en Europa continental se remonta al siglo XVI, la aparición de este idioma como herramienta de comunicación internacional no comenzó a ser palpable hasta el siglo XVIII. El siglo XX, sobre todo su segunda mitad, supuso la consolidación de este idioma como lengua franca de Europa y el desplazamiento progresivo de otras lenguas de prestigio, como el francés y el alemán. El propósito de este artículo es describir dicho proceso histórico y analizar los factores políticos, sociales y económicos que han convertido a esta lengua en el principal instrumento de comunicación internacional del viejo continente.
\end{abstract}

Palabras clave: Lengua franca, política lingüística, planificación lingüística, Unión Europea.

\section{English in Europe: Origin and Consolidation of a lingua franca}

Abstract: Although the teaching of English in mainland Europe dates back to the sixteenth century, the emergence of this language as a tool for international communication didn't become evident until the eighteenth century. The twentieth century, especially during the second half, was witness to the consolidation of English as a lingua franca in Europe as it gradually superseded other prestigious languages such as French and German. The purpose of this paper is to describe this historical process and analyze the political, social and economic factors that made this language the major tool for international communication on the old continent.

Key words: Lingua franca, language policy, language planning, European Union.

\section{PERSPECTIVA HISTÓRICA}

La enseñanza del inglés en Europa continental se remonta al siglo XVI (Truchot 2002: 7). Sin embargo, a finales de este siglo, la idea del idioma inglés como instrumento de comunicación internacional no era más que una esperanza de futuro incluso para los intelectuales ingleses más relacionados con el comercio internacional. Así al menos se desprende de las palabras de Richard Mulcaster (1582: 256), a la sazón Director de la Merchant Taylors' School de Londres: "Our English Tung [...] is of small reatch, it strecheth no further than this Iland of ours, naie not there over all". Con todo, el propio Mulcaster anticipa un futuro en el que el inglés sería la lengua universal del imperio que serviría de vínculo de unión entre los distintos individuos de una comunidad lingüística similar a la alcanzada en otra época por los romanos y, posteriormente, por los normandos (Ferguson 2003: 168). En este

\footnotetext{
Fecha de recepción: junio 2009.

Fecha de aceptación y versión definitiva: septiembre 2009.

2 Profesor Contratado Doctor, CES Felipe II, Universidad Complutense de Madrid;

$\triangle$ dvitores@cesfelipesegundo.com.
} 
sentido, tal como apuntaba Alexander Gill (1972: 86) en 1619, ninguna lengua era más apropiada que el inglés "to unify the speech of all peoples in one universal vocabulary".

A pesar de esta creencia, en el siglo XVII, una vez iniciada la colonización inglesa en el Caribe y el Norte de América, el inglés todavía no se consideraba una lengua de contacto útil (Wright 2004: 136). Era el conocimiento del francés y del italiano el que permitía al viajero de la época comunicarse con las élites educadas a lo largo y ancho del continente (ibídem). Por otra parte, el viajero que transitara por África, el Nuevo Mundo o las Indias, encontraría que el holandés y el español eran los idiomas más utilizados (Bailey 1991: 60-1). Al menos eso es lo que se desprende de los diarios escritos durante diez años por el poeta Richard Flecknoe en el transcurso de su viaje por Europa, Asia, África y América (Crystal 1997: 65).

La aparición del inglés como herramienta de comunicación internacional no comenzó a hacerse notar hasta el siglo XVIII, en el que aumentó considerablemente el optimismo sobre el valor del inglés fuera de Gran Bretaña (Bailey 1991: 98). No en vano, David Hume llegó a reprochar a Edward Gibbon su intención de redactar uno de sus ensayos en francés. Según el filósofo escocés, en el futuro, el inglés acabaría superando al francés como lengua franca universal, del mismo modo que el latín había reemplazado al griego en otra época (Hagène 1996: 105-6). Para él, la clave del éxito futuro de esta lengua residía, sin lugar a dudas, en América. Este cambio de mentalidad con respecto a la lengua inglesa por parte de los súbditos británicos hizo que, a mediados del siglo XVIII, el Duque de Chesterfield pudiera afirmar lo siguiente: "I have a sensible pleasure in reflecting upon the rapid progress which our language has lately made, and still continues to make, all over Europe". ${ }^{3}$ Este creciente interés por la lengua fue acompañado de numerosas victorias militares, de una potente expansión colonial, de un proceso de industrialización y de una reforma política en el interior de Gran Bretaña (Wright 2004: 137), que servirían de modelo para el resto de los países europeos y que afianzarían el inglés como lengua de acceso al conocimiento científico (Crystal 1997: 71-2).

De esta manera, la influencia limitada que el inglés había tenido en Europa desde el siglo XVI (Truchot 2002: 7) fue transformándose, poco a poco, en una consolidación real de este idioma como lengua franca de comunicación internacional a lo largo del siglo XIX.

A esto contribuyó, sin duda, el hecho de que esta visión eurocéntrica del avance del inglés era compartida por muchos estadounidenses. Así parece deducirse de la carta remitida en 1870 por John Adams al Presidente del Congreso de los Estados Unidos durante una misión diplomática en Europa, en la que proponía la creación de una Academia de la Lengua Americana:

In the last century, Latin was the universal language of Europe. Correspondence among the learned, and indeed among merchants and men of business, and the conversation of strangers and travellers, was generally carried on in that dead language. In the present century, Latin has been generally laid aside, and French has been substituted in its place, but has not yet become universally established, and, according to present appearances, it is not probable that it will. English is destined to be the next and succeeding centuries more generally the language of the world than Latin was in the last or French is in the

Citado en Crystal (1997: 65). 
present age. The reason of this is obvious, because the increasing population in America, and their universal connection and correspondence with all nations will, aided by the influence of England in the world, whether great or small, force their language into general use, in spite of all the obstacles that may be thrown in their way, if any such there should be. (Adams 1780)

La carta de Adams describe la percepción de universalidad de la lengua inglesa por parte de los hablantes nativos y perfila un futuro en el que este idioma acabaría imponiéndose al francés como lengua de comunicación internacional. Aunque, a juzgar por los numerosos documentos aportados por Richard W. Bailey (1991: 107), ese futuro era, cada vez más, una realidad del presente. Sirva, a modo indicativo, la opinión de un escritor publicada en la revista Gentelman's Magazine en 1829, en la que se expresaba del siguiente modo:

It is evident to all those who have devoted any portion of attention to the subject, that the English language would, if proper care were devoted to its advancement, stand an excellent chance of becoming more universally diffused, read, and spoken, than any other now is, or ever has been. In Europe, the study of it seems to be gradually spreading. In Germany, Russia, and Scandinavia it is esteemed an essential, in France a highly useful, branch of education; in Africa it is gradually superseding the Dutch, and becoming the medium of valuable information. In Australasia it is not only widely spoken, as the only European language known on that vast continent, but written and printed in an almost incredible number of newspapers, magazines, and reviews. In Asia so great is the desire manifested to learn it, that it was thought $[\ldots]$ that, if proper facilities were afforded, it would, in fifty years, supersede Hindoostanee, and become the court and camp language of India. In America, millions already speak, write, and read it as their mother tongue. [...] Never before did a language look forward to so bright a prospect as this, and nothing could retard its swift match but our own incredible folly. (ibídem: 107)

Tal como se desprende del texto anterior, la consolidación del inglés como lengua franca internacional era algo que se daba prácticamente por sentado ya en la primera mitad del siglo XIX y solo un descuido por parte de los Estados y organismos encargados de promocionar este idioma podría hacer que esta tendencia se invirtiera. Sin embargo, esta última opción no parecía probable si se tiene en cuenta la potente expansión colonial que dio lugar al Imperio Británico y, por extensión, a la expansión del inglés en el mundo. En palabras de un comentarista político en 1849:

As a vehicle of our institutions and principles and civil and religious liberty, it is belting the earth, pushing east and west, and extending over the five great geographical divisions of the world, giving no doubtful presage that, with its extraordinary resources for ameliorating the condition of man, it will soon become universal. (ibídem: 116)

Efectivamente, el inglés se estaba imponiendo en los lugares en los que la presencia colonial británica era un hecho. Esta imposición se realizó de dos formas diferentes, aunque ambas ligadas al espíritu colonial del Imperio Británico. En las áreas geográficas en las que la potencia colonial británica expulsó de sus tierras a los habitantes autóctonos, la lengua de dichos territorios pasó a ser el inglés. Este es el patrón que se siguió en América del Norte 
y Australasia, ${ }^{4}$ en donde la población autóctona que consiguió sobrevivir a la fuerza militar de la metrópoli fue aislada en las reservas o condenada a vivir en la marginalidad. Algo que, a la larga, significaba la pérdida de sus estructuras y referentes culturales y sociales (Wright 2004: 137). En otras áreas geográficas, como África y Asia, en las que los colonos británicos no expulsaron a la población autóctona sino que la emplearon como mano de obra en las plantaciones y las minas, el inglés era la lengua de la clase dirigente, al tiempo que la lengua de contacto entre esta y el resto de los habitantes. Esto tuvo como consecuencia la aparición, por una parte, de una burocracia integrada por un personal bilingüe $y$, por otra, de una especie de pidgin. En cualquier caso, en estas zonas geográficas, la introducción del inglés no significó la desaparición de las lenguas autóctonas, sino que la diglosia social permitió la convivencia de ambas (ibídem: 137).

En este contexto, debe decirse que el idioma de una potencia colonial supone la introducción de un nuevo instrumento de comunicación en la colonia en cuestión al tiempo que refleja los vínculos existentes entre la colonia y la metrópoli. Como ha apuntado Crystal (1997: 71), estos vínculos fueron especialmente importantes en el caso del inglés por la relevancia histórica del momento en el que se formaron, ya que facilitaron el acceso a una cultura que, más que ninguna otra, había sido responsable de la Revolución Industrial.

Efectivamente, Inglaterra fue el primer país que llevó a cabo toda una serie de transformaciones que la situaron en la vanguardia del progreso mundial. Los cambios en la agricultura, la población, los transportes y la tecnología favorecieron el desarrollo industrial. La industria textil algodonera fue el sector líder de la industrialización (Hobsbawm 1995: 91; Mathias 2001: 117) y la base de la acumulación de capital (Kindleberger 2006: 191) que abrió paso, en una segunda fase, a la siderurgia y al ferrocarril (Hartwell 1971: 124).

A mediados del siglo XVIII, la industria británica tenía unos cimientos sólidos y su expansión se proyectaba en dos ámbitos diferentes: las industrias de bienes de producción y de bienes de consumo (Mathias 2001: 12). Así, en Gran Bretaña se desarrolló plenamente el capitalismo industrial, lo que supuso la supremacía industrial británica hasta 1870 aproximadamente, así como también financiera y comercial desde mediados de siglo XVIII hasta la Primera Guerra Mundial (Kindleberger 2006).

Todo este progreso económico fue acompañado de un desarrollo tecnológico que permitió la racionalización de la producción manufacturera. Además, gran parte de los inventos que aparecieron durante este período fueron patentes inglesas (Mathias 2001: 35), lo que da fe de de su posición de liderazgo mundial.

Con todo, este progreso económico no habría sido posible sin la aparición de un contingente considerable de mano de obra barata motivado por la mejora de las condiciones de vida de la población. Dicha mejora favoreció una progresiva transición demográfica. Los nuevos alimentos venidos de América, como la patata, el tomate y el maíz, permitieron la introducción de una dieta más saludable. Además, a esta mejoría de la alimentación se sumaba el sostenimiento de la tasa de natalidad (Hudson 1992: 86). Asimismo, como la mortalidad disminuyó debido al fin de las crisis de alimentación, a los avances médicos y al descenso de la morbilidad de las enfermedades contagiosas y las epidemias catastróficas, la

\footnotetext{
4 Esta zona incluye Australia, Nueva Zelanda, Nueva Guinea y muchas islas pequeñas cercanas que forman la parte oriental de Indonesia.
} 
población aumentó considerablemente (Hobsbawm 1995: 36). Por otro lado, la edad fértil se adelantó, ya que los hombres y las mujeres no estaban sujetos a los ciclos de producción campesina y, al asegurarse los ingresos gracias al trabajo en la fábrica, podían casarse antes. Cambió, por tanto, el modelo de matrimonio europeo. Todos estos factores propiciaron este aumento de la población que, a lo largo del siglo XIX, llegó a cuadruplicarse en Inglaterra (Hudson 1992: 133) y que, en definitiva, supuso su rejuvenecimiento.

Por otra parte, el comercio recibió el impulso de la demanda exterior, que aún tenía una economía feudal y esclavista. Desde la firma de Tratado de Utrecht en 1713, desparecieron los privilegios proteccionistas del comercio de España con América. Esto supuso la libre competencia de los productos industriales ingleses con los autóctonos. Los productos industriales eran mucho más baratos $\mathrm{y}$, aunque de una calidad inferior, esta era lo suficientemente buena como para desplazar a la producción autóctona (Hobsbawm 1995: 93).

Las consecuencias lingüísticas de esta primacía económica y cultural de Gran Bretaña fueron enormes y dejaron su impronta en el ámbito económico, político, social y cultural de todos los países afectados por este proceso de industrialización (Crystal 2006: 426). La nueva terminología derivada del avance tecnológico y científico tuvo un impacto inmediato sobre la propia lengua inglesa, que incorporó miles de palabras a su léxico (Nevalainen y Van Ostande 2006: 303). Aunque quizá más importante desde el punto de vista de la primacía del inglés sobre el resto de las lenguas europeas fuera el hecho de que, al ser los países de habla inglesa los motores de la innovación tecnológica, todo el que quisiera acceder a este conocimiento para beneficiarse de él debía aprender bien el inglés (Crystal 1997: 72). Esto supuso, además, un intercambio de conocimientos basado en la lengua inglesa que se desarrolló en dos direcciones: por un lado, tanto empresarios como trabajadores acudieron a estos países para impregnarse de las nuevas técnicas de producción y, por otro lado, muchos británicos se desplazaron a otros países para enseñar los nuevos métodos. Todo ese intercambio de conocimiento utilizaba como canal principal de comunicación la lengua inglesa. No es por tanto sorprendente el hecho de que, si se suman la producción científica norteamericana y británica, entre 1750 y 1900 aproximadamente la mitad de la producción científica y tecnológica relevante estuviese redactada en inglés (ibídem: 73). Este dato es válido para la situación del inglés en el mundo. Sin embargo, si atendemos a la situación estrictamente europea, en la primera mitad del siglo XIX el francés seguía superando ampliamente al inglés como la lengua de referencia en el ámbito científico (Wright 2004: 139).

Por otro lado, el fuerte avance del inglés también estuvo ligado a la introducción de nuevas ideas en el ámbito político. El hecho de que Gran Bretaña fuera la cuna de la democracia parlamentaria, contribuyó a hacer que el aprendizaje del inglés fuese algo obligado para todos aquellos que quisieran tener acceso a estas nuevas ideas e hizo incorporar en las lenguas de destino palabras copiadas directamente del inglés (ibídem).

En el ámbito religioso, el inglés también fue una de las lenguas que permitieron el acceso a los conocimientos necesarios para alcanzar la salvación. Las misiones inglesas tuvieron una importancia vital para la expansión del inglés en el mundo. Tanto es así que, a mediados del siglo XIX, se publicaban y distribuían más biblias en inglés que en el resto de las lenguas habladas en los demás países en los que estaba asentado el cristianismo (ibídem). 
Con todo, la revolución industrial así como la divulgación de las ideas políticas y religiosas no habrían sido posibles sin la aparición de ciertos avances tecnológicos que representaron un papel determinante en la difusión de todos estos conocimientos. Así, la tecnología del vapor revolucionó el mundo de la impresión en papel posibilitando la publicación masiva de textos en inglés que iban desde manuales de instrucciones hasta periódicos de gran tirada. Además, a medida que estas innovaciones fueron introduciéndose en América del Norte, el número de publicaciones en inglés creció de manera exponencial (Crystal 1997: 73).

\section{LA INTRODUCCIÓN DEL INGLÉS COMO LENGUA FRANCA INSTITUCIONAL}

En suma, puede decirse que el siglo XIX supuso la sustitución del francés y del alemán por el inglés como lengua de comunicación internacional. Sin embargo, en el contexto europeo, para ver la primera aparición digna de mención del uso del inglés como lengua franca institucional habrá que esperar hasta 1919, año de la firma del Tratado de Versalles, que puso fin a la Primera Guerra Mundial (Truchot 1994: 142). De hecho, el propio Woodrow Wilson insistió en que los Tratados de Versalles, Sèvres y Trianon se publicasen tanto en inglés como en francés (Wright 2004: 143). Algo que parecía normal, dado que los representantes británicos y americanos que asistieron a las reuniones preparatorias no sabían francés, mientras que el delegado francés sí que sabía inglés. De este modo, la mayoría de las negociaciones se desarrollaron en inglés y, aunque el Tratado final fue redactado en inglés y francés, la versión francesa parecía una traducción de la inglesa (Truchot 1994: 142). Esta fue la primera vez que la posición del francés como lengua de redacción de los tratados fue desafiada (Wright 2004: 143). Aunque este acontecimiento no fue seguido de una difusión del inglés tan intensa como la que se produjo tras la Segunda Guerra Mundial, al menos sí significó la cesión del testigo del francés al inglés como lengua de comunicación internacional (Truchot 1994: 142). Al igual que ha ocurrido con otras lenguas en épocas pasadas, el comienzo del declive del francés como lengua utilizada en la diplomacia iba acompañado de la pérdida de influencia política y económica de su país de referencia: Francia (Wright 2004: 143).

El sistema introducido por la Sociedad de Naciones transfirió las antiguas colonias alemanas de África, Oriente Medio, Asia y Pacífico a la supervisión de los vencedores $\mathrm{y}$, de este modo, la influencia del idioma inglés aumentó considerablemente en las áreas controladas directamente por Gran Bretaña o por otras naciones de habla inglesa (Crystal 1997: 78). Una vez más, la lengua francesa sufrió un duro golpe al ser excluida de las preparaciones para la redacción del Pacto de la Sociedad de Naciones (Wright 2004: 143). Sin embargo, una vez establecida esta organización internacional, el francés pasó a ser, junto con el inglés, una de las dos lenguas oficiales. En lo que respecta al inglés, la Sociedad de Naciones fue la primera organización internacional que confirió un lugar especial al inglés en su funcionamiento interno. Algo que, por otra parte, parecía necesario, dado que el carácter multilingüe de esta alianza hacía especialmente útil la inclusión de una lengua franca que cada vez parecía más extendida (Crystal 1997: 79).

$\mathrm{Si}$ seguimos la lógica expuesta anteriormente, que vincula el auge de una lengua al poderío económico, político y militar de los Estados en los que se habla, la primacía del 
inglés debería haber iniciado una senda descendente tras la Segunda Guerra Mundial. Efectivamente, la resolución de la contienda coincide con el declive económico y político de Gran Bretaña, lo que hizo también que su ciencia y tecnología, que en otro tiempo fueran un acicate para el aprendizaje del inglés, pasase ahora a un segundo plano en la esfera internacional. Como ha sugerido Wright (2004: 140), el auge de la lengua inglesa era un fenómeno en gran medida elitista, ya que su uso se limitaba a una función auxiliar en el ámbito mercantil, administrativo y también como vehículo para acceder a las nuevas ideas políticas y científicas. Sin embargo, si se hubiese requerido otra lengua para acceder a estas fuentes, el inglés como lengua franca habría acabado desapareciendo. Si esta desaparición no se hizo efectiva fue, principalmente, porque el idioma de los Estados Unidos, la nueva potencia hegemónica en el terreno económico, político, militar y cultural, era también el inglés (ibídem: 141). Efectivamente, tras la Segunda Guerra Mundial, el inglés era la lengua de los vencedores. Las otras dos lenguas europeas que habían sido utilizadas recientemente como lenguas francas se estaban batiendo en retirada (Ammon 1996: 251). Por una parte, el alemán había perdido su papel como lengua de la ciencia y la tecnología. Asimismo, el francés había perdido gran parte de su prestigio como lengua de la diplomacia debido a la colaboración del gobierno de Vichy, lo que hizo que fuese relegada tanto en las negociaciones de posguerra como en la redacción de los tratados (Wright 2004: 143). De esta forma, la pérdida de prestigio de la lengua inglesa en Europa, derivada del progresivo desmantelamiento del imperio británico entre 1946 y 1997, ${ }^{5}$ fue compensada en gran medida por el afianzamiento en la escena internacional de los Estados Unidos, una nueva superpotencia que, como tal, también estaba interesada en la promoción de su lengua nacional como lengua franca internacional en el marco de un proceso de "neocolonialismo" (Fishman 1996: 5).

\section{LA CONSOLIDACIÓN DEL INGLÉS COMO LENGUA FRANCA EUROPEA}

Truchot (1994: 142) ha establecido dos períodos en el proceso de expansión del inglés en Europa desde el final de la Segunda Guerra Mundial. El primero abarcaría hasta finales de los años setenta. En este lapso de tiempo, el inglés se convirtió en la lengua dominante en la política internacional, los intercambios comerciales, la ciencia, la información, las producciones cinematográficas y televisivas y las canciones populares. Esta posición de dominio del inglés se puede atribuir, casi directamente, a la omnipresencia cultural de los Estados Unidos en Europa. El segundo período comprendería desde principios de los años ochenta en adelante, si bien en ciertas partes de Europa, como Suecia, Noruega, Finlandia y Holanda, el inicio de esta etapa fue anterior. Este período supuso la expansión y consolidación del inglés como lengua franca en la parte occidental del continente y, una vez iniciados los cambios políticos, también en Europa central y oriental.

Prueba de ello es la introducción progresiva del inglés en los sistemas educativos europeos. La rivalidad de la enseñanza del inglés con la del francés y el alemán en la educación secundaria se inició en la década de 1880, si bien el conocimiento de esta lengua ya era algo habitual en los círculos profesionales (Van Essen 1997: 99). En algunas partes de Alemania,

El proceso descolonizador británico se inicia con la independencia de Transjordania en 1946 (Louis 2007: 403-5) y finaliza con la entrega de Hong Kong en 1997 (Louis et ál. 2001: 667). Para una descripción detallada de este proceso descolonizador, véase McIntyre 1998. 
la enseñanza del inglés comenzó a tener preferencia sobre la de francés desde la década de los veinte en adelante. Así, Ammon (1996: 250) establece dos puntos cruciales que sirvieron de estímulo inmediato para el afianzamiento del inglés como lengua franca europea. El primero fue la introducción del inglés como primera lengua extranjera en los colegios alemanes en 1937 en lugar del francés, que hasta entonces había disfrutado de dicha posición. Aunque, probablemente, las razones de esta decisión fueron heterogéneas, no es aventurado suponer que las consideraciones raciales a favor de una lengua germánica en lugar de una románica ejercieran una gran influencia en su adopción bajo el liderazgo Nazi (ibídem). El segundo acontecimiento se produjo tras la Segunda Guerra Mundial, cuando varios países del norte de Europa rebajaron de categoría al alemán, al tiempo que elevaron la del inglés, como asignatura impartida en los colegios (ibídem). Esta es la evolución típica que siguieron la mayoría de las lenguas extranjeras enseñadas en otros países de Europa, que no pudieron hacer frente a un competidor tan potente como el inglés (ibídem: 251). Con todo, hasta la segunda guerra mundial, la enseñanza del inglés continuó siendo modesta en las regiones de Europa central y oriental, donde el alemán y el francés estaban firmemente establecidos. Tras la guerra, se desarrollaron dos tendencias sobre la incorporación del inglés en el ámbito educativo que coincidían con las áreas geográficas de Europa occidental y los denominados países de Europa del este. Dentro de Europa occidental, en los países escandinavos el inglés reemplazó al alemán y al francés a partir de la década de 1950 en adelante como el primer idioma extranjero enseñado y, en los Países Bajos, desde los años sesenta. Durante este período, la enseñanza del inglés en Francia comenzó a superar claramente a la del alemán. En España, el abandono progresivo del francés en beneficio del inglés se produjo a finales de los años setenta y principios de los ochenta y un poco más tarde en Portugal e Italia (Truchot 2002: 7). En la zona de Europa oriental la enseñanza del ruso se hizo obligatoria tras la segunda guerra mundial y siguió siéndolo hasta finales de los años ochenta. Al final del período estalinista, el inglés fue reintroducido en la enseñanza, junto con el alemán, que, al ser el idioma de la República Democrática Alemana, aun continuó siendo enseñado, sobre todo en Hungría, Polonia y Checoslovaquia (Fodor y Peluau 2003: 86). Por su parte, el francés, que todavía ocupaba un lugar destacado en los sistemas educativos de Rumanía y Bulgaria, vio como su posición se desplazaba gradualmente a favor del inglés (ibídem). La importancia del inglés en estos últimos países creció progresivamente hasta finales de la década de 1980 (Braine 2005: xv).

En Europa del Este, la obligatoriedad de aprender ruso en las escuelas quedó sin efecto a partir de 1990, permitiendo así la competición con otras lenguas europeas para disputarse la posición de segunda lengua en dicha área geográfica. Esto benefició claramente al inglés (Truchot 2002: 8) y, en cualquier caso, redujo la gama de lenguas en liza a únicamente tres: el inglés, el francés y el alemán (Fodor y Peluau 2003: 90). Por otra parte, en países como Hungría, Polonia y la República Checa, el fuerte asentamiento del alemán como segunda lengua aún representaba un gran obstáculo para la penetración del inglés como lengua extranjera. Sirva como ejemplo el caso de Hungría en el que los datos relativos al aprendizaje de lenguas extranjeras aportados por el Ministerio de Educación configuran el siguiente gráfico: 
Gráfico 1.

\section{Evolución del número de alumnos de Hungría que estudia una lengua extranjera distinta del ruso}

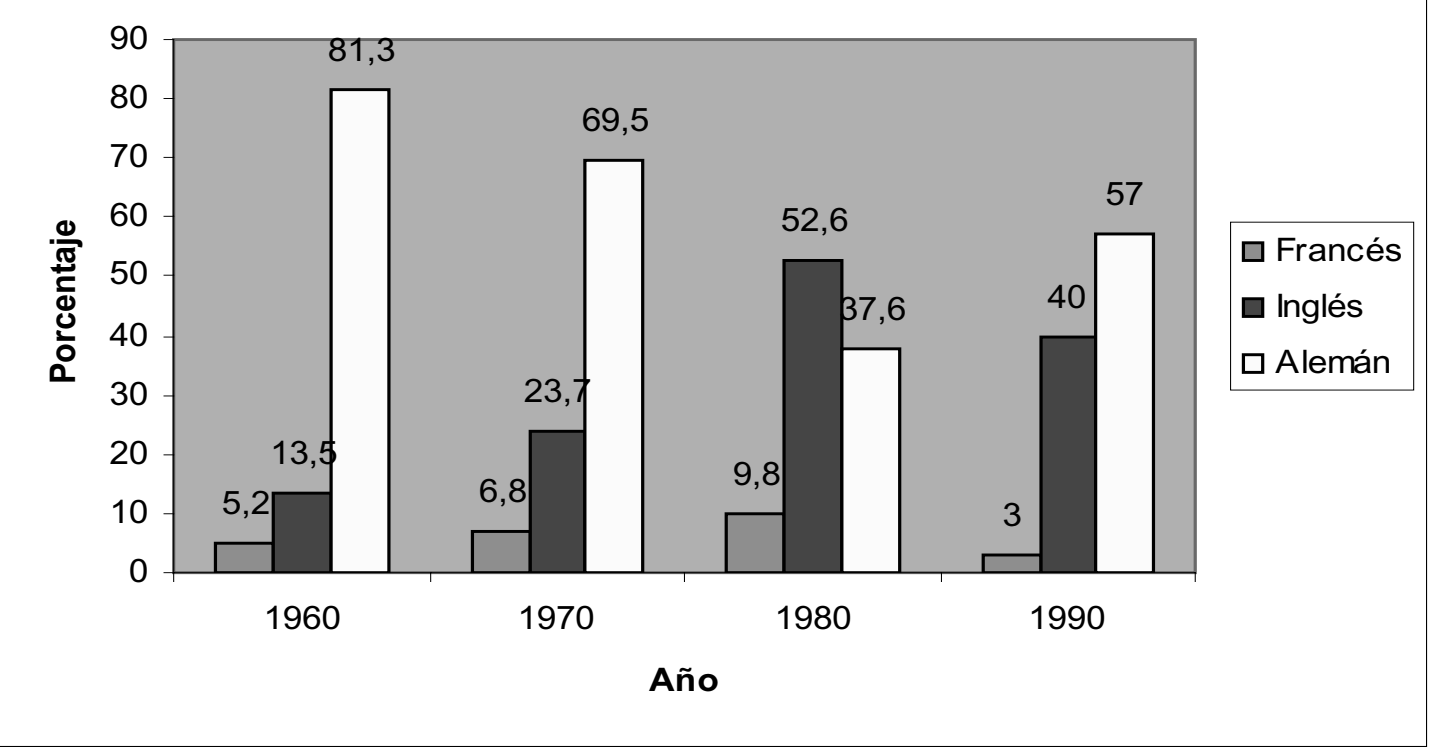

Fuente: Ministerio de Educación de Hungría (citado en Fodor y Peluau 2003: 89)

Como puede observarse, el gráfico muestra un ascenso imparable en el aprendizaje del inglés con respecto al francés y al alemán desde 1960 hasta 1980. Posteriormente, esta tendencia se invierte a favor del alemán, de forma que este idioma supera ampliamente al inglés en el número de alumnos que lo estudia. La explicación ha de buscarse en dos factores principales. Por una parte, la reunificación alemana sirvió como estímulo para reivindicar de nuevo la posición del alemán en los países donde esta lengua estaba más asentada. Por otra parte, la desmembración de la Unión Soviética provocó un trasvase de alumnos del ruso hacia otras lenguas europeas (Mikhalchenko y Trushkova 2003: 271). De hecho, con Rusia en medio del desplome político y financiero, la percepción sobre la utilidad del ruso en la esfera internacional se había deteriorado sustancialmente, debido en parte a la escasa inversión en la promoción de esta lengua (ibídem: 285). Por el contrario, en Europa del Este, el alemán aun se percibía como una lengua fuerte dentro de las instituciones europeas y a Alemania como el motor económico de Europa capaz de hacer frente al desafío económico que suponía la reunificación. Con este telón de fondo, la racionalidad individual de los húngaros invitaba a escoger el aprendizaje del alemán, una lengua que, por tradición y cercanía, les resultaba mucho más familiar que el inglés y cuyo aprendizaje, casi con total seguridad, podrían rentabilizar posteriormente en el mercado común europeo.

Uno de los elementos que sin duda supuso un espaldarazo para la consolidación del inglés como primera lengua extranjera en los sistemas educativos de los Estados europeos fue el hecho de que este idioma fuese adoptado como instrumento de comunicación, en algunos casos de forma exclusiva, por los principales organismos internacionales. 
Aparte de la Unión Europea que, al menos de iure, respeta el multilingüismo integral, el inglés es una de las lenguas vehiculares de muchas otras organizaciones internacionales en las que el régimen jurídico es más restrictivo. Así, el inglés es una de las seis lenguas oficiales de la ONU, junto con el árabe, el chino, el español, el francés y el ruso. ${ }^{6}$ Este régimen lingüístico se cumple en la mayoría de los documentos oficiales, que son traducidos a estos seis idiomas, así como en las reuniones oficiales, que disponen de interpretación en dichas lenguas. Sin embargo, en algunas dependencias, las conferencias y los documentos de trabajo solo se redactan en francés e inglés - o en español, francés e inglés- y las publicaciones se realizan en estos dos o tres idiomas. No es de extrañar, por tanto, que la propia organización haya mostrado su preocupación por el fuerte avance del inglés en detrimento de las otras lenguas oficiales. ${ }^{7}$

Otras organizaciones menos multiculturales que la ONU y con un objetivo más concreto han sido incluso más restrictivas en la adopción de su régimen lingüístico. Este es el caso de la OTAN que, aunque sus Tratados fundacionales fueron redactados en inglés y francés, siempre ha utilizado el inglés como lengua de trabajo debido a la fuerte influencia de los Estados Unidos (Wright 2000: 87).

Esta tendencia hacia el uso del inglés también puede observarse en otras organizaciones internacionales esencialmente europeas como son la Organización para la Seguridad y la Cooperación en Europa (OSCE) y, en mayor medida, el Consejo de Europa. Esta última organización estableció desde un principio como lenguas oficiales de deliberación y de archivo de documentos oficiales (ibídem: 88) el inglés y el francés. Algo que resulta explicable si se tiene en cuenta cuáles fueron los diez países que firmaron el Tratado de Londres el 5 de mayo de 1949. El Consejo de Europa acepta, sin embargo, la utilización de otras lenguas pero normalmente dicho uso debe ser apoyado económicamente (Petschen Verdaguer 1997: 83). El hecho de que su sede esté situada en Estrasburgo ha favorecido el uso del francés en las oficinas de esta institución. Sin embargo, entre los delegados que asisten a la Asamblea y entre los juristas del Tribunal, la tendencia ha sido utilizar el inglés como lengua de comunicación (Wright 2000: 88) confirmando así la afirmación realizada por algunos autores (Petschen Verdaguer 1997: 83) de que, en el futuro, el francés tendría un papel muy secundario.

\section{CONCLUSIONES}

El estudio de la evolución histórica del inglés en Europa muestra un patrón en cierto modo identificable con la suerte seguida por el francés y, en menor medida, el alemán: la existencia de fenómenos extralingüísticos de peso que favorecen la utilización de esta lengua fuera del grupo de hablantes nativos para convertirse en una lengua franca (Wright: 2004: 118). En este sentido, los cambios políticos, económicos, culturales y sociales ocurridos durante el siglo XIX supusieron la sustitución del francés y del alemán por el inglés como lengua de comunicación internacional. En el contexto europeo propiamente dicho, el punto de inflexión de esta sustitución coincide con la firma en 1919 del Tratado de Versalles,

\footnotetext{
6 La base legal para el establecimiento de estas lenguas oficiales es el artículo 111 de la Carta de las Naciones Unidas de 26 de junio de 1945.

$7 \quad$ Véase Linguamón 2007.
} 
que puso fin a la Primera Guerra Mundial (Truchot 1994: 142) y significó el comienzo del declive del francés como lengua utilizada en la diplomacia. La Segunda Guerra Mundial dio carta de naturaleza a esta nueva posición del inglés y supuso el inicio de una fuerte expansión en Europa, beneficiada en gran medida por el establecimiento de los Estados Unidos como primera potencia mundial. Los años de la posguerra fueron testigo de la consolidación del inglés como lengua de la política y el comercio internacionales, así como de la cultura mundial y la educación. En Europa, esto se hizo patente mediante la introducción progresiva del estudio del inglés como lengua extranjera en los centros educativos de los distintos países, lo que supuso una pérdida de protagonismo del francés y del alemán, que hasta entonces ocupaban una posición de liderazgo en este ámbito.

\section{REFERENCIAS BIBLIOGRÁFICAS}

Adams, J. 1780. "A Letter to the President of Congress: Proposal for an American Language Academy". http://ourworld.compuserve.com/homepages/JWcrawford/ Adams.htm (21 Abril 2009)

Ammon, U. 1996. "The European Union (EU - formerly European Community). Status Change of English during the last 50 years". Ed. J. A. Fishman. Post-imperial English: Status Change in Former British and American Colonies, 1940-1990. Berlín: Mouton de Gruyter. 241-270.

BaILey, R. W. 1991. Images of English: A Cultural History of the Language. Ann Arbor, Michigan: University of Michigan Press.

Braine, G. 2005. "Introduction”. Teaching English to the World: History, Curriculum, and Practice. Ed. G. BRAINE. New York: Routledge. xi-xx.

Carta de las Naciones Unidas de 26 de junio de 1945 en San Francisco. http://www. un.org/spanish/aboutun/charter/index.htm (1 agosto 2008)

CRYSTAL, D. 1997. English as a global language. Cambridge: Cambridge University Press.

. 2006. "English Worldwide". A history of the English language. Eds. R. M. Hogg y D. Denison. Cambridge: Cambridge University Press. 420-439.

Ferguson, M. W. 2003. Dido's Daughters: Literacy, Gender, and Empire in Early Modern England and France. Chicago and London: University of Chicago Press.

Fishman, J. A. 1996. "Introduction: Some Empirical and Theoretical Issues". Postimperial English: Status Change in Former British and American Colonies, 19401990. Ed. J. A. Fishman. Berlín: Mouton de Gruyter. 3-12.

Fodor, F. y S. Peluau. 2003. Language geostrategy in eastern and central. Europe: Assessment and perspectives. Languages in a Globalising World. Eds. J. MAURAIS y M. A. Morris. Cambridge: Cambridge University Press. 85-98.

Gill, A. 1972 (1619). Logonomia Anglica (1619). Vol 2. Estocolmo: Almqvist \& Wiksell. 
HaGÈGE, C. 1996. Le français, histoire d'un combat. Boulogne-Billancourt: Michel Hagège.

Hartwell, R. M. 1971. The Industrial Revolution and Economic Growth. London: Methuen.

Hobsbawm, E. J. 1995. En torno a los orígenes de la Revolución Industrial. Madrid: Siglo Veintiuno Editores.

Hudson, P. 1992. The Industrial Revolution. London: Oxford University Press.

Kindleberger, C. P. 2006. A Financial History of Western Europe. United Kingdom: Taylor \& Francis.

LinguAmón. 2007. "La ONU declara 2008 el Año Internacional de las Lenguas". Linguamón, 17 May. http://www10.gencat.net/pres_casa llengues/AppJava/frontend/ noticies detall.jsp?id=106\&idioma $=6$ ( 23 octubre 2008$)$.

Louis, W. R. 2007. Ends of British Imperialism: The Scramble for Empire, Suez, and Decolonization: Collected Essays. London: I. B. Tauris.

. et ál. 2001. Oxford History of the British Empire: Historiography. vol. 5. Oxford: Oxford University Press.

Mathias, P. 2001. The First Industrial Nation: The Economic History of Britain 17001914. Londres: Routledge.

McIntyre, W. D. 1998. British Decolonization, 1946-1997: When, Why and How Did the British Empire Fall? New York: St. Martin's Press.

Mikhalchenko, V. I y Y. V. Trushrova. 2003. "Russian in the modern world". Languages in a Globalising World. Eds. J. Maurais y M. A. Morris. New York: Cambridge University Press. 260-290.

Mulcaster, R. 1582. The First Part of the Elementarie which Entreateth Chefelie of the Right Writing of our English Tung. London: Thomas Vautroullier.

Nevalainen, T. y I. Tieken-Boon Van Ostande 2006. "Standardisation". A history of the English language. Eds. R. M. Hogg y D. Denison. Cambridge: Cambridge University Press. 271-311.

Petschen Verdaguer, S. 1997. "Los principios reguladores de las lenguas en la Europa Contemporánea”. Revista de antropología social 6, 73-88. http://www.ucm.es/ BUCM/revistas/cps/1131558x/articulos/RASO9797110073A.PDF (3 septiembre 2008)

Truchot, C. 1994. "The spread of English in Europe". Journal of European Studies 24, 141-151. de Europa.

. 2002. Key Aspects of the Use of English in Europe. Estrasburgo: Consejo

Van Essen, A. 1997. "English in Mainland Europe - A Dutch Perspective". World Englishes 16, 1: 95-103. 
Wright, S. 2000. Community and communication. Clevedon: Multilingual Matters. . 2004. Language policy and language planning: From nationalism to globalisation. Basingstoke: Palgrave. 